\title{
Foramina of the anterior mandible in dentate and edentulous mandibles
}

\author{
K. Natsis ${ }^{1}$, E. Repousi ${ }^{2}$, I. Asouhidou ${ }^{1}$, Ch. Siskos ${ }^{3}$, A. Ioannidi ${ }^{3}$, M. Piagkou ${ }^{2}$ \\ ${ }^{1}$ Department of Anatomy, School of Medicine, Faculty of Health Sciences, Aristotle University of Thessaloniki, Greece \\ 2Department of Anatomy, School of Medicine, Faculty of Health Sciences, National and Kapodistrian University \\ of Athens, Greece \\ ${ }^{3}$ Department of Endodontics, Dental School, National and Kapodistrian University of Athens, Greece
}

[Received: 30 August 2015; Accepted: 3 September 2015]

The study provides a morphometric analysis of the foramina located at the anterior mandible according to dental status. The inner surface from the midline to the distal border of the second premolars of 70 dentate and 27 edentulous Greek adult dry mandibles was investigated. The lingual foramina were divided into medial and lateral foramina. Foramina located at the alveolar process and the midline were subdivided according to their location to genial tubercles. Moreover, the height of the mandible in the genial symphysis and the distances from the foramina to the alveolar crest and the lower border of the mandible were measured. Medial and lateral lingual foramina were presented in $97.9 \%$ and $78.4 \%$ of the mandibles, respectively. The alveolar medial and lateral lingual foramina were detected in 19.6\% and $27.3 \%$, respectively. The mean height of the genial symphysis was $32.06 \pm$ $\pm 4.88 \mathrm{~mm}$ for the dentate and $23.87 \pm 5.37 \mathrm{~mm}$ for the edentulous mandibles. The meticulous knowledge of the topography of the lingual foramina and their content is of paramount importance for dentists, oral and maxillofacial surgeons during dental implants placement. Middle and lateral lingual foramina are constant structures, while the alveolar foramina presented only in dentate mandibles. The foramina location is directly affected by dental status. The morphology of edentulous mandibles increases the risk of intraoperative complications at the anterior mandible. (Folia Morphol 2016; 75, 2: 204-210)

Key words: alveolar process, mandible, dental implants, lingual foramina, resorption

\section{INTRODUCTION}

A plethora of accessory foramina in various locations of the external and the inner surface of the mandible are described [27], but are more frequently presented on the inner surface of the anterior mandible [4]. Foramina localised in the midline (area of the genial symphysis), superior, medial and inferior to the genial tubercles (GT) are called medial lingual foramina (MLF) [18], while those situated laterally to them, are named lateral lingual foramina (LLF) and vary in number and location. The foramina situated at the alveolar part have been described separately as nutrient foramina $[1,3]$. In the current study, the latter foramina are named medial and lateral alveolar foramina, as Przystańska and Bruska [24] mentioned. The meticulous knowledge of the distribution and content of the MLF and LLF is of crucial importance for dentists, oral and maxillofacial surgeons, when intervening in the anterior mandible for dental implants placement and genioplastic or grafting procedures. 
The MLF may contain a branch of the sublingual artery or vein [22] or a branch of the submental artery or an anastomotic branch of both the sublingual arteries $[6,15,17,18,21]$. Moreover, branches of the mylohyoid [22] or the lingual nerve may pass through $\operatorname{MLF}[17,27]$. The MLF superior to the GT may contain branches of the lingual artery, vein and nerve and the MLF inferior to GT may give passage to branches of the mylohyoid nerve and submental and/or sublingual arteries [13]. Perforating branches, when present, emerge from a sublingual-sublingual anastomosis or a branch of the submental artery may pass through LLF $[15,21]$. Nerve fibres for the supplementary innervation of the anterior mandibular teeth may also pass through LLF [7].

Morphometry and shape of the foramina of the mandible are affected by the teeth existence or absence [2]. These alterations in mandible are the result of the alveolar process resorption that occurs after teeth loss. Various local (mechanical factors, chronic inflammatory periodontal disease and trauma) and systemic (nutritional, metabolic and hormonal) factors play a role in morphology of the mandible $[2,11]$. The current study was designed to assess the topography and morphometry of the lingual foramina (LF) and distribution of the mandibular height in the midline in Greeks. A different evaluation of these parameters was conducted to dentate and edentulous mandibles. Moreover, clinical aspects related to the foramina presence and edentulism are also discussed.

\section{MATERIALS AND METHODS}

Ninety-seven Greek adult dry mandibles from the osseous collection of our Departments were investigated. Mandibles of children, mandibles damaged, and bones with pathological conditions were excluded. In adults, the main factor that affects the morphology of the mandible is the teeth presence or absence [11]. Considering this parameter, all mandibles were subdivided according to the dental status into dentate and edentulous mandibles. Seventy dentate mandibles with apparent alveolar process and teeth in the anterior area and 27 edentulous mandibles with a variable degree of atrophy of the alveolar process and no teeth in the anterior area were observed (Fig. 1).

The inner surface between the midline and the distal border of the second premolars was examined on both sides for the foramina presence. The foramina were identified only if a flexible wire (diameter $0.2 \mathrm{~mm}$ ) could be passed through them.

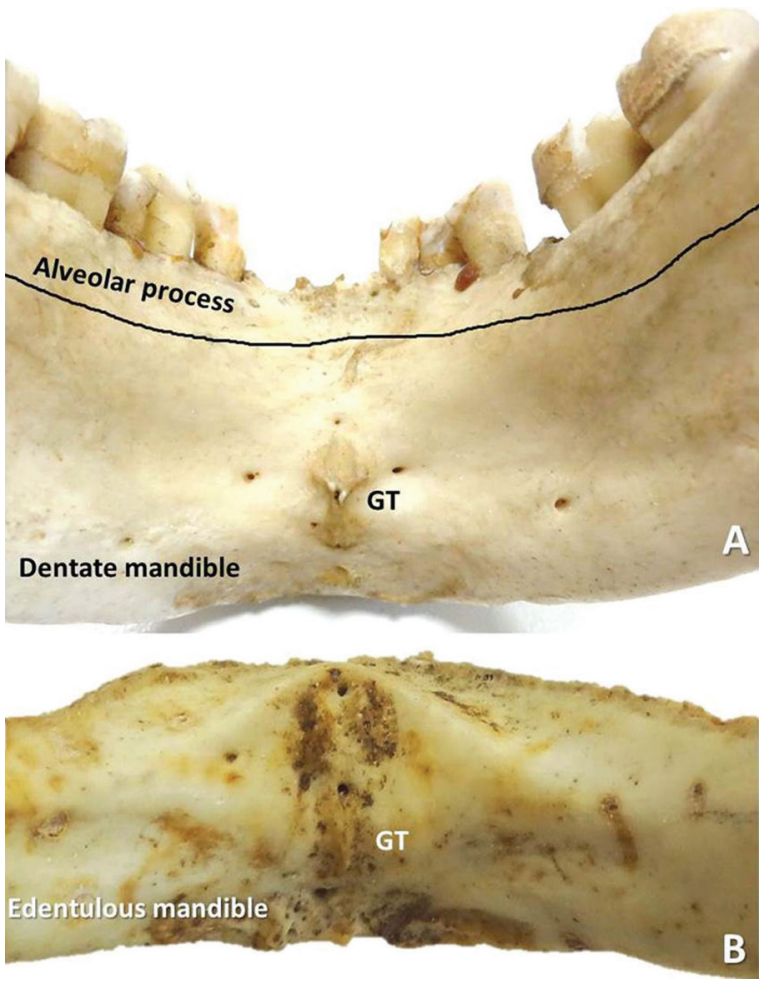

Figure 1. A. Dentate mandible with the anterior dentition and the alveolar process; B. An edentulous mandible with a high grade of resorption; GT — genial tubercles.

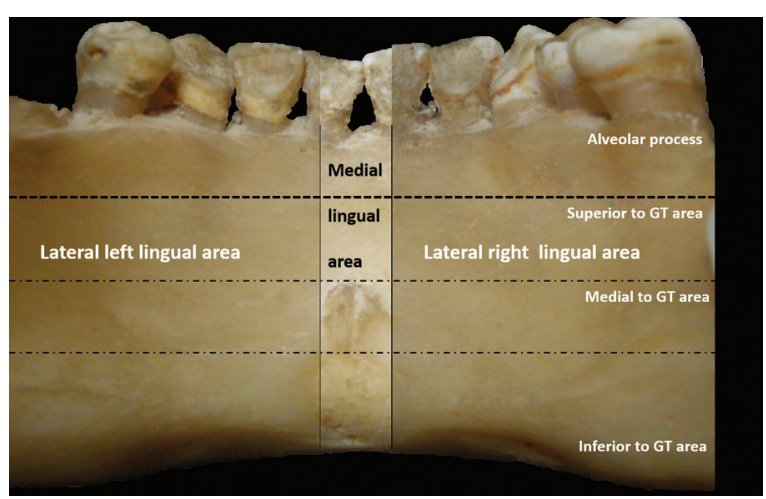

Figure 2. Area of the genial tubercles (GT) is named medial lingual area (bright part) and areas lateral to GT are the lateral right and left lingual areas (darker part). These regions were subdivided according to the alveolar process as alveolar medial and lateral lingual areas. The remaining mandibular area was further subdivided using GT as a landmark to superior to GT, medial to GT and inferior to GT areas.

The exact location of GT was identified and using the tubercles as osseous landmark, the body of the mandible was subdivided in further areas for a detailed investigation of the presence, number and location of the LF. Foramina that were positioned superior, medial and inferior to GT are nominated as MLF, 

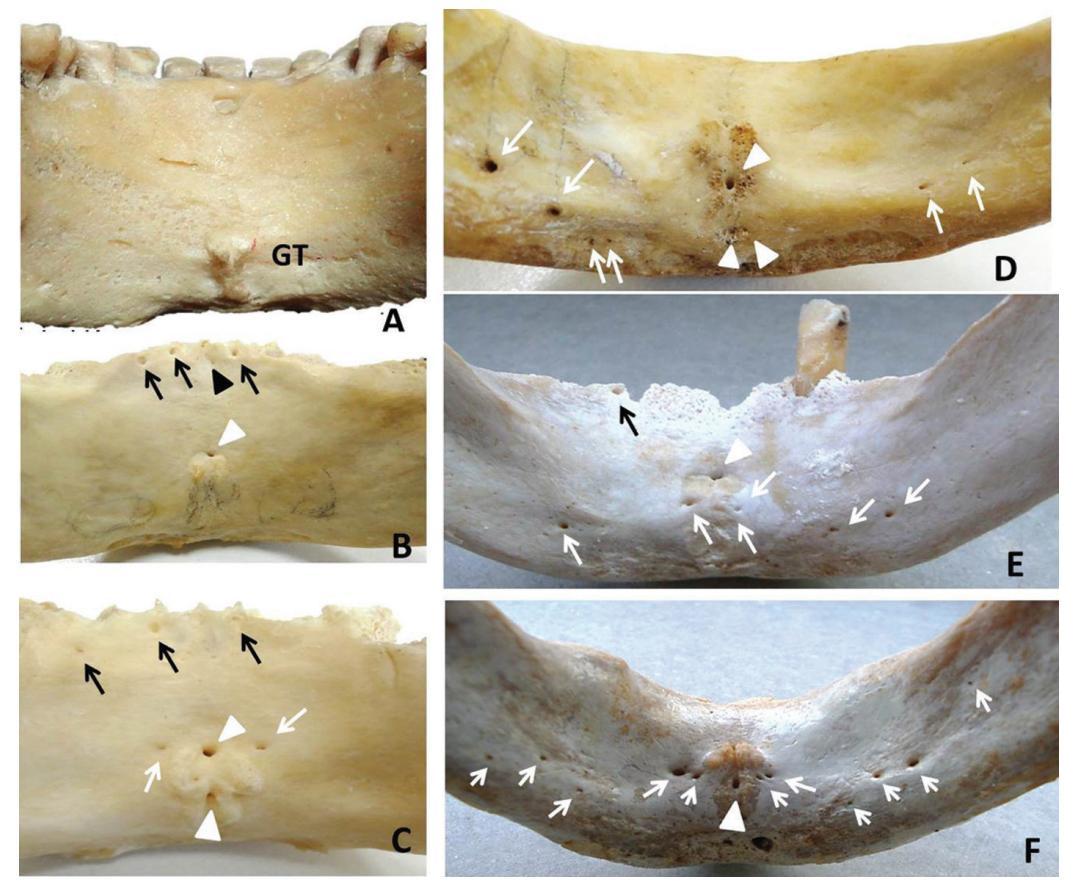

Figure 3. A. Dentate mandible with no foramina. The genial tubercles (GT) are shown; B. Black triangle indicates the alveolar medial foramen (MLF) and black arrows show the lateral lingual foramina (LLF). White triangle indicates the MLF superiorly to GT; C. Black arrows indicate the alveolar LLF and white arrows the LLF. The white triangles show the MLF medially and inferiorly to GT; $\mathbf{D}$. The white triangles indicate the MLF (medial and inferior to GT areas) and white arrows the LLF; E. Multiple foramina (alveolar — black arrow, MLF — white triangle, LLF — white arrows) in a mandible; F. A MLF inferiorly to GT (white triangle) and multiple LLF (white arrows) are shown in an edentulous mandible.

while any other foramen localised laterally to these regions is reported as LLF. The upper border of the area superior to GT was considered as the inferior part of the alveolar process. Foramina that were located at the alveolar process are classified as alveolar MLF and LLF (Fig. 2). Finally, the height of the mandible at the genial symphysis (distance from the alveolar crest - AC to the lowest point of the mandibular border - LBM) and the exact location of the MLF and LLF were identified by measuring the distances AC-foramen and LBM-foramen. All measurements were performed using a Mitutoyo digital Vernier calliper accurate to $0.01 \mathrm{~mm}$. Descriptive statistics (mean \pm \pm standard deviation, minimum and maximum) were found. Correlations between the foramen existence and the side of occurrence were analysed using the Fisher's exact test. For the correlation of the symphyseal height regarding the foramen existence and the dental status, the t-test was used. The t-test was also performed for the correlation of the MLF distances according to the dental status. The Pearson correlation was used for the correlations between the genial symphysis and the distances AC-MLF and LBM-MLF. Statistical analysis was performed using SPSS Software for Windows version 21.0.

\section{RESULTS}

Ninety-five (97.2\%) mandibles presented at least one foramen, while $2(2.06 \%)$ dentate mandibles had no foramina (Fig. 3). No mandible existed with LLF without having at least one MLF. The MLF and LLF presence is summarised Table 1. The number of MLF ranged from 0 to 4, LLF ranged from 0 to 7 and total foramina ranged from 0 to 12 . The majority of the mandibles presented 1-2 MLF and 1-3 LLF. The strong correlation existed between the right and left-sided LLF ( $p=0.001)$, indicating either the simultaneous presence of the LLF number on both sides or their absence. No difference in the MLF and LLF presence was found in dentate and edentulous mandibles. The alveolar MLF and LLF were presented only in dentate mandibles (Fig. 3). As regards the correlations between the foramina location at the genial symphysis, the only statistically significant correlation was found between the superior and medial to GT areas $(p=0.001)$, indicating the existence of a foramen exclusively at one of the two areas. Any correlation between the height of the genial symphysis and the other variables were conducted only for the MLF, as all measured distances corresponded in the same vertical level. Contrariwise, 
Table 1. The incidence of the appearance of the medial and lateral lingual foramina according to their location to the anterior area of the mandible

\begin{tabular}{lccc}
\hline Foramina & Medial lingual foramina & \multicolumn{2}{c}{ Lateral lingual foramina } \\
\cline { 2 - 3 } location & Number $-\mathbf{n}(\%)$ & Right side & Left side \\
\hline Alveolar crest & $19(19.6 \%)$ & $23(23.7 \%)$ & $30(30.9 \%)$ \\
Superior to GT & $75(77.3 \%)$ & $2(2.1 \%)$ & $1(1 \%)$ \\
Medial to GT & $33(34 \%)$ & $23(23.7 \%)$ & $28(28.9 \%)$ \\
Inferior to GT & $60(61.9 \%)$ & $28(28.9 \%)$ & $26(26.8 \%)$ \\
Total & $95(97.2 \%)$ & $59(60.8 \%)$ & $70(72.2 \%)$ \\
\hline
\end{tabular}

GT - genial tubercles

Table 2. Descriptive statistics of the distances from the lingual foramina (LF) to the alveolar crest (AC), the genial tubercles (GT) and the lower border of the mandible (LBM) in dentate mandibles. The $r$ and $p$ coefficient correspond to the results of Pearson correlation; SD — standard deviation

\begin{tabular}{|c|c|c|c|c|c|}
\hline Distances & Mean \pm SD & Minimum & Maximum & $r$ & $p$ \\
\hline From $\mathrm{AC}$ to the foramen superior to the GT & $18.30 \pm 3.97$ & 9.01 & 26.59 & 0.831 & 0.001 \\
\hline From LBM to the foramen superior to the GT & $18.28 \pm 3.20$ & 10.96 & 24.08 & 0.641 & 0.001 \\
\hline From $\mathrm{AC}$ to the foramen medial to the GT & $22.34 \pm 7.67$ & 7.09 & 37.06 & 0.770 & 0.001 \\
\hline From LBM to the foramen medial to the GT & $14.37 \pm 3.76$ & 8.21 & 21.28 & -0.002 & 0.992 \\
\hline From $\mathrm{AC}$ to the foramen inferior to the GT & $26.77 \pm 6.38$ & 11.57 & 40.50 & 0.809 & 0.001 \\
\hline From LBM to the foramen inferior to the GT & $8.21 \pm 4.52$ & 0.02 & 29.19 & 0.101 & 0.286 \\
\hline From $A C$ to the lateral LF right & $24.50 \pm 4.45$ & 15.29 & 34.58 & - & - \\
\hline From LBM to the lateral LF right & $12.37 \pm 4.74$ & 5.17 & 24.08 & - & - \\
\hline From $A C$ to the lateral LF left & $23.39 \pm 5.76$ & 6.84 & 35.32 & - & - \\
\hline From LBM to the lateral LF left & $12.31 \pm 5.41$ & 2.77 & 24.51 & - & - \\
\hline From right lateral LF to the GT & $13.60 \pm 6.74$ & 2.59 & 28.18 & - & - \\
\hline From left lateral LF to the GT & $12.39 \pm 6.80$ & 1.90 & 26.73 & - & - \\
\hline Height of the genial symphysis & $32.06 \pm 4.88$ & 21.26 & 43.23 & - & - \\
\hline
\end{tabular}

no further analysis was performed for the LLF, as they corresponded in different vertical levels. The height of the genial symphysis at the edentulous mandibles was $23.87 \pm 5.37 \mathrm{~mm}$ (min: 15.56, max: 37.97), and it was statistically significantly lower ( $p=0.001)$ compared to the dentate mandibles (32.06 $\pm 4.88 ; 21.26-43.23$, respectively) $\mathrm{mm}$. The mandibular height in the midline in the whole sample was 29.23 $6.37(15.56-43.23) \mathrm{mm}$.

Mandibles that presented alveolar MLF were higher $(p=0.001)$ versus those that had no such foramina. The MLF did not show any correlation. An extensive statistical analysis was performed as regards the AC-MLF and LBM-MLF distances, separately for the dentate and edentulous mandibles (Tables 2, 3).
Since the alveolar MLF were presented only in dentate mandibles, the correlations between the two groups could not be performed and thus they were excluded from the analysis. The t-test was conducted for comparisons of the corresponding measurement between dentate and edentulous groups and all distances at the edentulous group were statistically significantly lower at the 0.01 level. The lower values of distances in edentulous mandibles indicate the bone resorption on AC and LBM. The Pearson's linear correlation was used in assessing the correlation between AC-MLF and LBM-MLF and the height of the genial symphysis (Tables 2, 3). A positive linear correlation between the symphyseal height and the AC-MLF distance was detected in both groups. As 
Table 3. Descriptive statistics of the distances from the lingual foramina (LF) to the alveolar crest (AC), the genial tubercles (GT) and the lower border of the mandible (LBM) in edentulous mandibles. The $r$ and $p$ coefficient correspond to the results of Pearson correlation; SD — standard deviation

\begin{tabular}{lccccc}
\hline Distances & Mean \pm SD & Minimum & Maximum & r & p \\
\hline From AC to the foramen superior to the GT & $8.74 \pm 4.11$ & 1.06 & 15.57 & 0.828 & 0.001 \\
From LBM to the foramen superior to the GT & $16.21 \pm 2.40$ & 12.17 & 23.71 & 0.342 & 0.013 \\
From AC to the foramen medial to the GT & $12.06 \pm 4.90$ & 6.89 & 17.63 & 0.888 & 0.002 \\
From LBM to the foramen medial to the GT & $14.58 \pm 5.64$ & 9.64 & 23.88 & 0.873 & 0.053 \\
From AC to the foramen inferior to the GT & $18.81 \pm 4.99$ & 11.37 & 27.46 & 0.904 & 0.001 \\
From LBM to the foramen inferior to the GT & $6.54 \pm 2.18$ & 2.55 & 10.36 & -0.038 & 0.086 \\
From AC to the lateral LF right & $17.34 \pm 5.81$ & 3.86 & 28.48 & - & - \\
From LBM to the lateral LF right & $9.73 \pm 5.66$ & 12.65 & 20.01 & - & - \\
From AC to the lateral LF left & $16.54 \pm 5.76$ & 5.91 & 25.68 & - & - \\
From LBM to the lateral LF left & $8.80 \pm 4.22$ & 1.06 & 15.77 & - & - \\
From right lateral LF to the GT & $13.04 \pm 7.66$ & 1.52 & 26.27 & - & - \\
From left lateral LF to the GT & $11.08 \pm 6.23$ & 1.24 & 20.93 & - & - \\
\hline Height of the genial symphysis & $23.87 \pm 5.37$ & 15.56 & 37.97 & - & - \\
\hline
\end{tabular}

regards the LBM-MLF distance, the only significant correlation was found for the distances from LBM to foramina situated superior to GT. The correlations between the symphyseal height and AC-MLF are stronger compared with LBM-MLF, in both groups.

\section{DISCUSSION}

Since placement of dental implants in the anterior mandible has been widely applied, the meticulous knowledge of the foramina topography and their content is of paramount importance for dentists and maxillofacial surgeons. In our research, the presence of at least one MLF indicates that the MLF is a constant structure, similarly to other studies [13, 18, 26]. A negative correlation between the MLF presence in the areas superior and medial to GT was found, indicating that usually a single foramen exists at the level of GT and above. This finding indicating that the most frequent location of the MLF was at the level of GT and above highlights the clinical significance of the MLF location during dental implants placement $[13,23,26]$. The LLF were detected in $78.4 \%$ of the mandibles $(60.8 \%$ on the right and $72.2 \%$ on the left side) in our study. The LLF occurrence presents a wide range from $30 \%$ to $76 \%[4,10,13,23]$ among several studies, due to the different methodology in their topography determination. In our study, the LLF were observed at the level of GT and below, similarly to Liang et al. [13] and Przystańska and Bruska [23].
In our sample, all mandibles that presented LLF had at least one MLF. The LLF are bilateral structures [4, 23] and our findings strongly support this view. As a result, clinicians should be aware for this simultaneous existence. The diameters of the MLF range from $0.7 \pm$ $\pm 0.3 \mathrm{~mm}$ to $1.01 \pm 0.4 \mathrm{~mm}[5,28]$ and these foramina lead to bony canals with a downward or an upward direction $[10,28]$. The LLF are smaller than MLF, their diameters range from $0.6 \pm 0.3 \mathrm{~mm}$ to $0.93 \pm 0.31 \mathrm{~mm}$ $[5,28]$ and their bony canals run upward $[10]$.

After tooth loss, the alveolar bone is affected by a continuous bone resorption $[8,12]$. As a result, we examined separately the foramina in the alveolar process. The alveolar MLF were detected in 19.6\% of the mandibles and the alveolar LLF in $23.7 \%$ on the right and $30.9 \%$ on the left side. These foramina were observed only in dentate mandibles, as during the process of the alveolar bone resorption, they reach the $A C$ and may even be lost. The alveolar MLF and LLF are nutrient foramina, through which pass interalveolar branches from the mandibular and incisive arteries that enter the mucous membrane in the AC [3] or may contain a neurovascular bundle [1].

Dentate mandibles differ significantly from edentulous mandibles [19] also in our investigation, particularly in the symphyseal area, as all the measured distances presented a statistically significant difference between the two groups. In dentate mandibles, 
the mean symphyseal height was $32.6 \pm 4.88 \mathrm{~mm}$, while the height in edentulous mandibles was particular lower in $23.87 \pm 5.37 \mathrm{~mm}$. The AC-MLF distance differed between dentate and edentulous mandibles, due to the horizontal pattern of resorption both from the labial and lingual aspects [11]. As regards the MLF in the area superior to GT, which is the most clinically significant, the AC-MLF in dentate group was $18.30 \pm$ $\pm 3.97 \mathrm{~mm}$, while in edentulous group was $8.74 \pm$ $\pm 4.11 \mathrm{~mm}$. In edentulous mandibles, the MLF in the area superior to GT was in close proximity to the AC. In addition, the LBM-MLF in edentulous mandibles was statistically significantly lower than in dentate mandibles. This result indicates that the LBM is also affected after the tooth loss. Kingsmill and Boyde [11] reported a similar finding after the investigation of the mandibular height at the mental foramen. In our study, the MLF-LBM in the area superior to GT was measured in $18.28 \pm 3.20 \mathrm{~mm}$ in dentate group and in $16.21 \pm 2.40 \mathrm{~mm}$ in edentulous mandibles, similarly to Yildirim et al. [29] and contrariwise to Liang et al. [13] and Rosano et al. [26] who found the distance to be $10.6 \pm 5.5 \mathrm{~mm}$ and $12.5 \pm 2.1 \mathrm{~mm}$. The LLF were located more inferiorly in the mandible with a mean AC-LLF distance of $24.50 \pm 4.45 \mathrm{~mm}$ (right-sided LLF) and $23.39 \pm 5.76 \mathrm{~mm}$ (left-sided LLF) and a mean LLF-GT $13.60 \pm 6.74 \mathrm{~mm}$ (right-sided LLF) and $12.39 \pm 6.80 \mathrm{~mm}$ (left-sided LLF).

The detailed knowledge of the above distances is of immense significance considering the content of the MLF and LLF. The anterior mandible is supplied in order of frequency by the sublingual artery (branch of the lingual or the facial artery) [21], the submental artery (branch of the facial artery) [21] or both the sublingual and submental arteries [9]. The sublingual artery is in high risk of laceration and transection when courses horizontally between the sublingual fossa and the mandible $[9,15]$. The injury of the submental artery may occur when its branches enter the sublingual space perforating the mylohyoid muscle or take a roundabout course near the mandibular surface [9]. The sublingual artery enters through the lingual canal at an average height of $10.3 \mathrm{~mm}$ from the LBM [15] and its diameter and the average blood flow are sufficient to cause a life-threatening haemorrhage $[15,16,26]$. A non-serious endosseous bleeding may also occur after the injury of the rich intraosseous vascular plexus formed from the LF canals [25]. The surgeon intervening in the anterior mandible should be particularly cautious in cases of edentulism due to the completely different osseous morphology of the mandible [12] and its arterial pattern. The horizontal bone loss and the projection of mental tubercles lead to lingual inclination of edentulous mandible and thus the drilling angulation should be adapted [8]. The life-threatening haemorrhage in the floor of the mouth occurred in a drilling depth of $\geq 15 \mathrm{~mm}$ [8] and considering the close proximity of the foramina to the $A C$ in edentulous mandibles, the implants length (especially long implants $>13 \mathrm{~mm}$ ) should be well considered [26]. As regards the arterial pattern alterations, the tooth loss resulting in a shift from a centrifugal to a centripetal blood circulation and the preservation of involved tissues is essential in edentulous mandibles [8]. Due to the decrease of mandibular height, the mylohyoid muscle reaches the $A C$ and the intervening surgeon apart from the arteries and structures located in the sublingual space should be alert of those below the mylohyoid muscle $[8,9]$. Another complication related to the content of MLF and LLF, caused during dental implants placement, is the sensory disturbances. This complication occurs after direct (perforation through the bony canal) or indirect (pressure by a haematoma) trauma, and/or after chronic stimulation (implant malposition and nerve stimulation) [14]. The above complications may be avoided if a preoperative diagnostic control is performed using the cone-beam computed tomography scan [20] and Doppler sonography [16].

Finally, a finding of our study is that the mandibles with alveolar MLF were higher versus those that had no such foramina. A possible explanation is that the dentate group included mandibles with a complete anterior and incomplete posterior dentition and thus a grade of resorption may have existed. As regards the results of Pearson correlation, we concluded that even though resorption existed at the LBM, this resorption was of a lower grade compared with the obvious atrophy at the AC.

\section{CONCLUSIONS}

In conclusion, the MLF and LLF are constant structures for dentate and edentulous mandibles. The alveolar foramina presented only in dentate mandibles. The location of MLF and LLF is directly affected by the dental status and in edentulous mandibles the MLF and LLF almost reach the AC. Possible intraoperative complications may occur and particularly edentulous patients are in higher risk. 


\section{REFERENCES}

1. Britt GN (1977) A study of human mandibular nutrient canals. Oral Surg Oral Med Oral Pathol, 44: 635-645.

2. Chrcanovic BR, Abreu MH, Custódio AL (2011) Morphological variation in dentate and edentulous human mandibles. Surg Radiol Anat, 33: 203-213.

3. Ennis LRM (1937) Roentgenographic variation of the maxillary sinus and the nutrient canals of the maxilla and the mandible. Int J Orthod Oral Surg, 23: 173-193.

4. Fanibunda K, Matthews JN (1999) Relationship between accessory foramina and tumour spread in the lateral mandibular surface. J Anat, 195: 185-190.

5. Gahleitner A, Hofschneider U, Tepper G, Pretterklieber M, Schick S, Zauza K, Watzek G (2001) Lingual vascular canals of the mandible: evaluation with dental CT. Radiology, 220: 186-189.

6. Hofschneider U, Tepper G, Gahleitner A, Ulm C (1999) Assessment of the blood supply to the mental region for reduction of bleeding complications during implant surgery in the interforaminal region. Int J Oral Maxillofac Implants, 14: 379-383.

7. Jeyaseelan N, Sharma JK (1984) Morphological study of unnamed foramina in north Indian human mandibles and its possible role in neurovascular transmission. Int J Oral Surg, 13: 239-242.

8. Kalpidis CD, Setayesh RM (2004) Hemorrhaging associated with endosseous implant placement in the anterior mandible: a review of the literature. J Periodontol, 75: 631-645.

9. Katsumi Y, Tanaka R, Hayashi T, Koga T, Takagi R, Ohshima H (2013) Variation in arterial supply to the floor of the mouth and assessment of relative hemorrhage risk in implant surgery. Clin Oral Implants Res, 24: 434-440.

10. Kawai T, Asaumi R, Sato I, Yoshida S, Yosue T (2007) Classification of the lingual foramina and their bony canals in the median region of the mandible: cone beam computed tomography observations of dry Japanese mandibles. Oral Radiol, 23: 42-48.

11. Kingsmill VJ, Boyde A (1998) Variation in the apparent density of human mandibular bone with age and dental status. J Anat, 192: 233-244.

12. Kingsmill VJ (1999) Post-extraction remodeling of the adult mandible. Crit Rev Oral Biol Med, 10: 384-404.

13. Liang $X$, Jacobs R, Lambrichts I, Vandewalle G (2007) Lingual foramina on the mandibular midline revisited: a macroanatomical study. Clin Anat, 20: 246-251.

14. Liang X, Lambrichts I, Corpas L, Politis C, Vrielinck L, Guo Wu MA, Jacobs R (2008) Neurovascular disturbance associated with implant placement in the anterior mandible and its surgical implications: literature review including report of a case. CJDR, 11: 56-64.

15. Loukas M, Kinsella CR Jr, Kapos T, Tubbs RS, Ramachandra S (2008) Anatomical variation in arterial supply of the man- dible with special regard to implant placement. Int J Oral Maxillofac Surg, 37: 367-371.

16. Lustig JP, London D, Dor BL, Yanko R (2003) Ultrasound identification and quantitative measurement of blood supply to the anterior part of the mandible. Oral Surg Oral Med Oral Pathol Oral Radiol Endod, 96: 625-629.

17. Madeira MC, Percinoto C, das Graças M, Silva M (1978) Clinical significance of supplementary innervation of the lower incisor teeth: a dissection study of the mylohyoid nerve. Oral Surg Oral Med Oral Pathol, 46: 608-614.

18. McDonnell D, Reza Nouri M, Todd ME (1994) The mandibular lingual foramen: a consistent arterial foramen in the middle of the mandible. J Anat, 184: 363-369.

19. Merrot O, Vacher C, Merrot S, Godlewski G, Frigard B, Goudot P (2005) Changes in the edentate mandible in the elderly. Surg Radiol Anat, 27: 265-270.

20. Naitoh M, Hiraiwa Y, Aimiya H, Ariji E (2009) Observation of bifid mandibular canal using cone-beam computerized tomography. Int J Oral Maxillofac Implants, 24: 155-159.

21. Nakajima K, Tagaya A, Otonari-Yamamoto M, Seki K, Araki K, Sano T, Okano T, Nakamura M (2014) Composition of the blood supply in the sublingual and submandibular spaces and its relationship to the lateral lingual foramen of the mandible. Oral Surg Oral Med Oral Pathol Oral Radiol, 117: 32-38.

22. Przystańska A, Bruska M (2010) Accessory mandibular foramina: histological and immunohistochemical studies of their contents. Arch Oral Biol, 55: 77-80.

23. Przystańska A, Bruska M (2012) Anatomical classification of accessory foramina in human mandibles of adults, infants, and fetuses. Anat Sci Int, 87: 141-149.

24. Przystańska A, Bruska M (2005) Foramina on the internal aspect of the alveolar part of the mandible. Folia Morphol, 64: 89-91.

25. Romanos GE, Gupta B, Crespi R (2012) Endosseous arteries in the anterior mandible: literature review. Int J Oral Maxillofac Impl, 27: 90-94.

26. Rosano G, Taschieri S, Gaudy JF, Testori T, Del Fabbro M (2009) Anatomic assessment of the anterior mandible and relative hemorrhage risk in implant dentistry: a cadaveric study. Clin Oral Implants Res, 20: 791-795.

27. Sutton RN (1974) The practical significance of mandibular accessory foramina. Aust Dent J, 19: 167-173.

28. Von Arx T, Matter D, Buser D, Bornstein MM (2011) Evaluation of location and dimensions of lingual foramina using limited cone-beam computed tomography. J Oral Maxillofac Surg, 69: 2777-2785.

29. Yildirim YD, Güncü GN, Galindo-Moreno P, Velasco-Torres $M$, Juodzbalys G, Kubilius M, Gervickas A, Al-Hezaimi K, Al-Sadhan R, Yilmaz HG, Asar NV, Karabulut E, Wang HL, Tözüm TF (2014) Evaluation of mandibular lingual foramina related to dental implant treatment with computerized tomography: a multicenter clinical study. Implant Dent, 23: 57-63. 